\title{
Optimization of extraction and characterization of Urtica dioica L. leaf extract for the evaluation of dose-dependent response in experimental hypertension
}

\author{
UNA-JOVANA VAJIĆ ${ }^{1, *}$ \\ ${ }^{1}$ University of Belgrade, Institute for Medical Research, Dr Subotića 4, 11129 Belgrade, Serbia \\ *Corresponding author: unajovana@imi.bg.ac.rs
}

Received: October 4, 2018

Accepted: October 5, 2018

Published on-line: December 20, 2018

Published: December 25, 2018

\begin{abstract}
This doctoral dissertation was focused on the optimization of the extraction parameters to achieve the maximum yield of phenolic compounds in the Urtica dioica L. leaf extracts. The extract with the highest content of phenolic compounds was tested in the model of experimental hypertension to determine its effects on oxidative and hemodynamic status, lipid profile, and biochemical parameters. It was shown that the most abundant phenolic compounds present in extracts of Urtica dioica L. were chlorogenic acid, 2-O-caffeoyl malic acid, and rutin. It was shown that aqueous-methanol is a better solvent for the extraction of phenolic compounds compared to aqueous-ethanol and water. Also, extracts prepared using ultrasound-assisted extraction technique had higher phenolic yields and antioxidant capacities than extracts obtained by maceration. The maximal content of phenolic compounds in the Urtica dioica L. leaf extracts was achieved by ultrasound-assisted extraction with $54 \%$ aqueous-methanol and $38 \mathrm{~min}$ extraction time. It was shown that the methanol percentage in the extraction solvent significantly affects phenolic yield, unlike the extraction time. Results of in vivo study showed that four-week long treatment with Urtica dioica L. leaf extract decreased arterial pressure and improved systemic oxidative status of spontaneously hypertensive rats, as well as the lipid status by increasing plasma HDL cholesterol and decreasing atherosclerosis-index.
\end{abstract}

Key words: optimization of extraction, stinging nettle, Urtica dioica, phenolic compounds, hypertension, oxidative stress

\author{
ABBREVIATIONS \\ SHR, spontaneously hypertensive rats \\ UE, Urtica dioica L. leaf extract \\ eNOS, iNOS, and nNOS endothelial, inducible, and neuronal nitric- \\ oxide synthase, respectively \\ $\mathrm{TF}$, total flavonoid content \\ HDL, high-density lipoprotein \\ LDL, low-density lipoprotein
}

\section{INTRODUCTION}

Modern life is characterized by numerous risk factors for cardiovascular diseases - the leading cause of mortality in the human population. These factors include behavioral risk factors such as irregular diet, lack of physical activity, obesity, stress, smoking, and etc. On the other hand, hypertension, another risk factor for cardiovascular diseases, affects one billion people worldwide and is being considered responsible
NOTE: Results from this dissertation have been published previously in:

Vajic, U.-J., Grujic-Milanovic, J., Miloradovic, Z., Jovovic, Dj., Ivanov, M., Danijela, K., Katarina, S., Bugarski, B., Mihailovic-Stanojevic, N., (2018). Urtica dioica L. leaf extract modulates blood pressure and oxidative stress in spontaneously hypertensive rats. Phytomedicine, 46, pp. 39-45.

Vajić, U.-J., Grujić-Milanović, J., Živković, J., Šavikin, K., Gođevac, D., Miloradović, Z., Bugarski, B.,

Mihailović-Stanojević, N., (2015). Optimization of extraction of stinging nettle leaf phenolic compounds using response surface methodology. Ind Crops Prod, 74, pp. 912-917. 
for $45 \%$ of deaths caused by heart diseases and $51 \%$ deaths as a consequence of stroke. Hypertension, combined with behavioral risk factors, as well as atherosclerosis and metabolic risk factors (e.g. diabetes) is the leading cause for the development and progression of cardiovascular diseases. Over the past few decades, fruit and vegetable-rich diet have been the focus of scientific interest because of its potential to reduce the risk of cardiovascular diseases.

Many components of this food have been proven to be beneficial for cardiovascular health, especially phenolic compounds. Ever since "French paradox", fruit and vegetable-rich dietrelated positive effects on human health have been largely attributed to phenolic compounds, especially flavonoids. There is an increasing body of evidence that long-term dietary intake of flavonoid-rich food has favorable effects on numerous chronic age-related diseases, especially cardiovascular diseases.

There are numerous plants rich in phenolic compounds. One of them is Urtica dioica L., commonly known as stinging nettle, a perennial herbaceous cosmopolitan plant of a substantial pharmacological significance. Since ancient times, different parts of Urtica dioica L. have been used in the treatment of numerous health problems. Today, herb and the roots are commonly used, root in the treatment of benign prostate hyperplasia, and herb for rheumatic conditions and urinary tract infections. In addition to phytotherapeutical use, few scientific studies have shown that Urtica dioica L. extracts exhibit in vivo hypotensive, diuretic, as well as vasorelaxant effects in isolated blood vessels. Moreover, this plant has the agricultural and industrial significance too, because it is a lowrequirement plant and a rich source of phenolic compounds and textile fibers. The majority of phenolic compounds detected in Urtica dioica L. leaf are caffeic acid derivates and glycosides of flavonols (usually quercetin an isorhamnetin glycosides). Some of them, such as rutin, quercetin, and chlorogenic acid has been shown to have cardioprotective effects. Therefore, identification and quantification of Urtica dioica L. phenolic compounds, maximization of their yield in the extract, as well as the potential application of the phenolrich Urtica dioica L. leaf extracts as a dietary supplement, is a promising research topic.

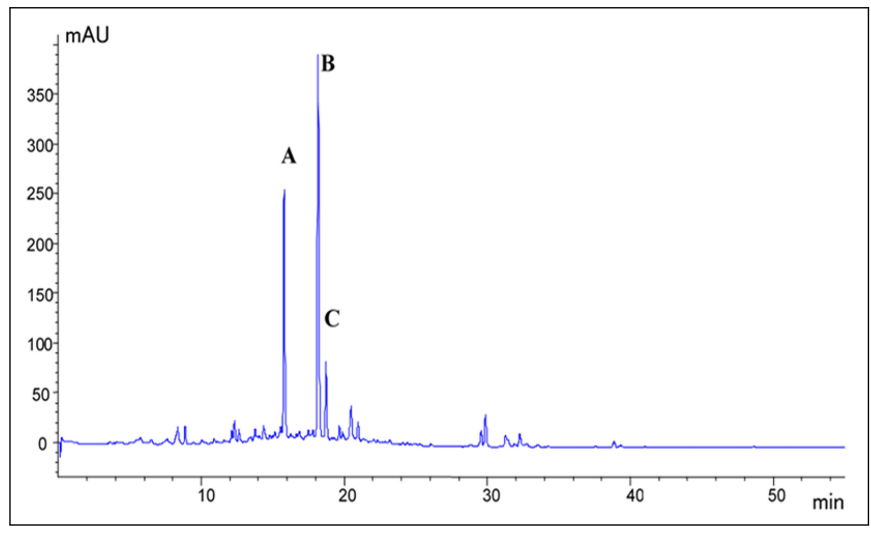

Fig. 1. Chromatogram of $50 \%$ methanolic leaf extract of Urtica dioica L. (A) chlorogenic acid, (B) 2-O-caffeoyl malic acid and (C) rutin (Published in Vajić et al., 2015)

\section{MATERIAL AND METHODS}

In the first phase of the optimization, preliminary experiments were conducted in order to determine extraction solvents for the further optimization. For this purpose, we used 50, 75 and $100 \%$ methanol, 50, 75 and $96 \%$ ethanol, and water for $30 \mathrm{~min}$ long maceration. The selection of solvent was made comparing total phenolic content (TP), contents of individual phenolic compounds, and antioxidant capacity of the extracts. In the second phase of the optimization, a full factorial design (two factors and three levels) and RSM (Response Surface Methodology) were used for determination of extraction conditions necessary to achieve maximal TP yield, as well as to determine the relationship between independent factors and the dependent variable. Independent factors considered were the percentage of alcohol in the solvent (X1) and extraction time (X2), while the dependent variable was TP content. Besides TP content in the extracts, individual phenolic compounds were identified and quantified using LC/MS and HPLC-DAD methods. Extracts were prepared by maceration. A secondorder polynomial equation was used for fitting experimental data and predicting the response. In the third phase, we examined the influence of the ultrasound on the yield of phenolic compounds in the Urtica dioica L. leaf extracts, since it has been shown before that ultrasound increases the efficiency of extraction by increasing the yield and by shortening the extraction time. Therefore, we used previously determined optimal extraction solvent and varying extraction times (10, 20,30 , and optimal extraction time) for ultrasound extraction of Urtica dioica L. leaf. The effects of ultrasound on the extraction of phenolic compounds were determined by comparison of TP content, total flavonoid (TF) content, contents of individual phenolic compounds, and antioxidant capacity of the extracts prepared using ultrasound with extracts prepared by maceration. The extract with the highest TP content was then lyophilized and used in in vivo part of the study.

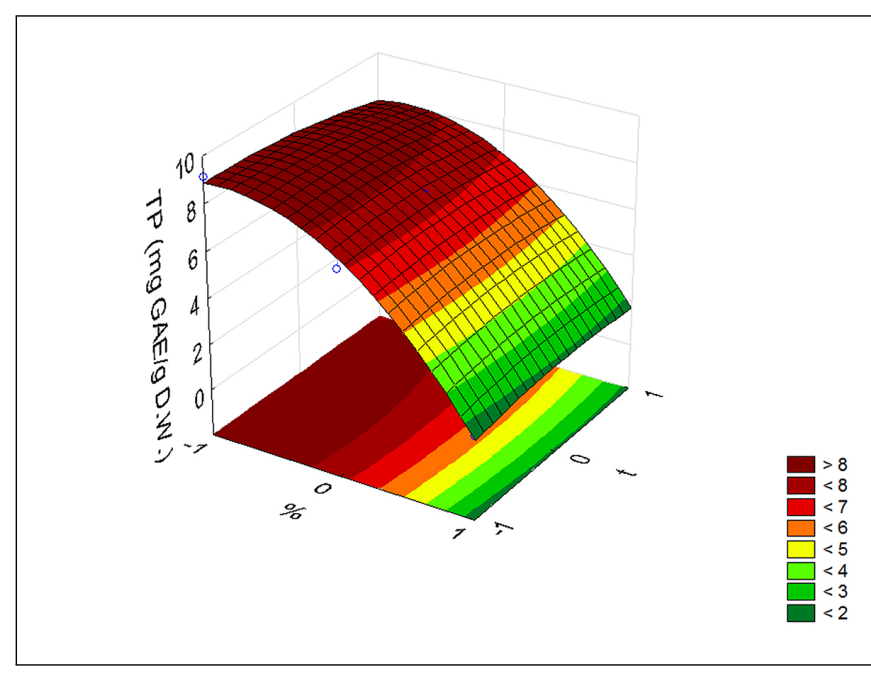

Fig. 2. Response surface plot showing the influence of the methanol percentage $(\%)$ and extraction time $(\mathrm{t})$ on total phenolic (TP) content in the Urtica dioica L. leaf extract (Published in Vajić et al., 2015)

In the second part of the study, we examined the effects of four-week long supplementation with three doses of $U r$ tica dioica L. leaf extract (UE) with the highest content of phenolic compounds in the experimental model of essential hypertension. Spontaneously hypertensive rats (SHR) were divided into five experimental groups which dally received by oral gavage 10,50 , and $200 \mathrm{mg} / \mathrm{kg} /$ day of UE and 10 $\mathrm{mg} / \mathrm{kg} /$ day of losartan. After the treatment, hemodynamic parameters, parameters of oxidative status, as well as biochemical parameters were measured. We measured in anesthetized animals systemic hemodynamic parameters such as systolic, diastolic, mean arterial pressure, cardiac output, heart rate, stroke volume, total peripheral vascular resistance, as well as regional hemodynamic parameters - flow and resistance 


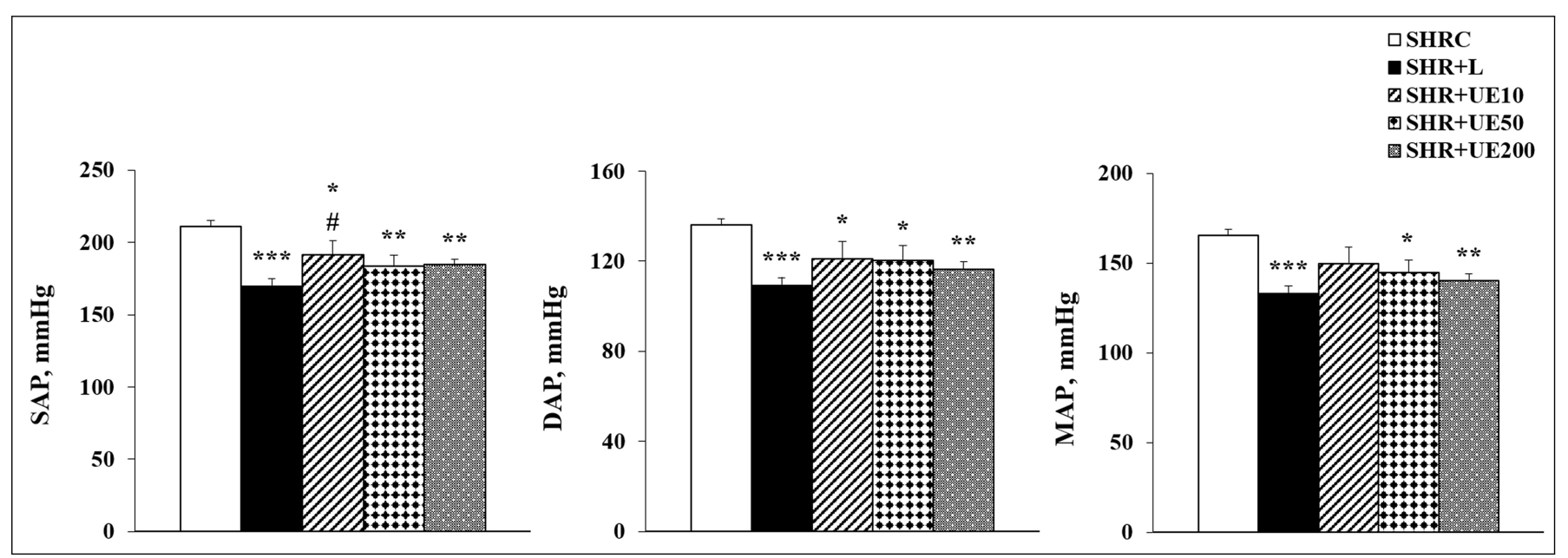

Fig. 3. Effects of four-week long daily supplementation with Urtica dioica L. leaf extract (UE) on systolic arterial pressure (SAP), diastolic arterial pressure (DAP), and mean arterial pressure (MAP) of SHR (Published in Vajic et al., 2018); SHRC received 0.5 mL/day of water; SHR+L received $10 \mathrm{mg} / \mathrm{kg} /$ day of losartan; SHR+UE10, SHR+UE50, and SHR+UE200 received 10, 50, and $200 \mathrm{mg} / \mathrm{kg} /$ day of UE, respectively; ; $<<0.05$, ${ }_{* *} \mathrm{p}<0.01$, and ${ }^{* * *} \mathrm{p}<0.001$ vs SHRC; $\# \mathrm{p}<0.05$ vs SHR $+\mathrm{L}$

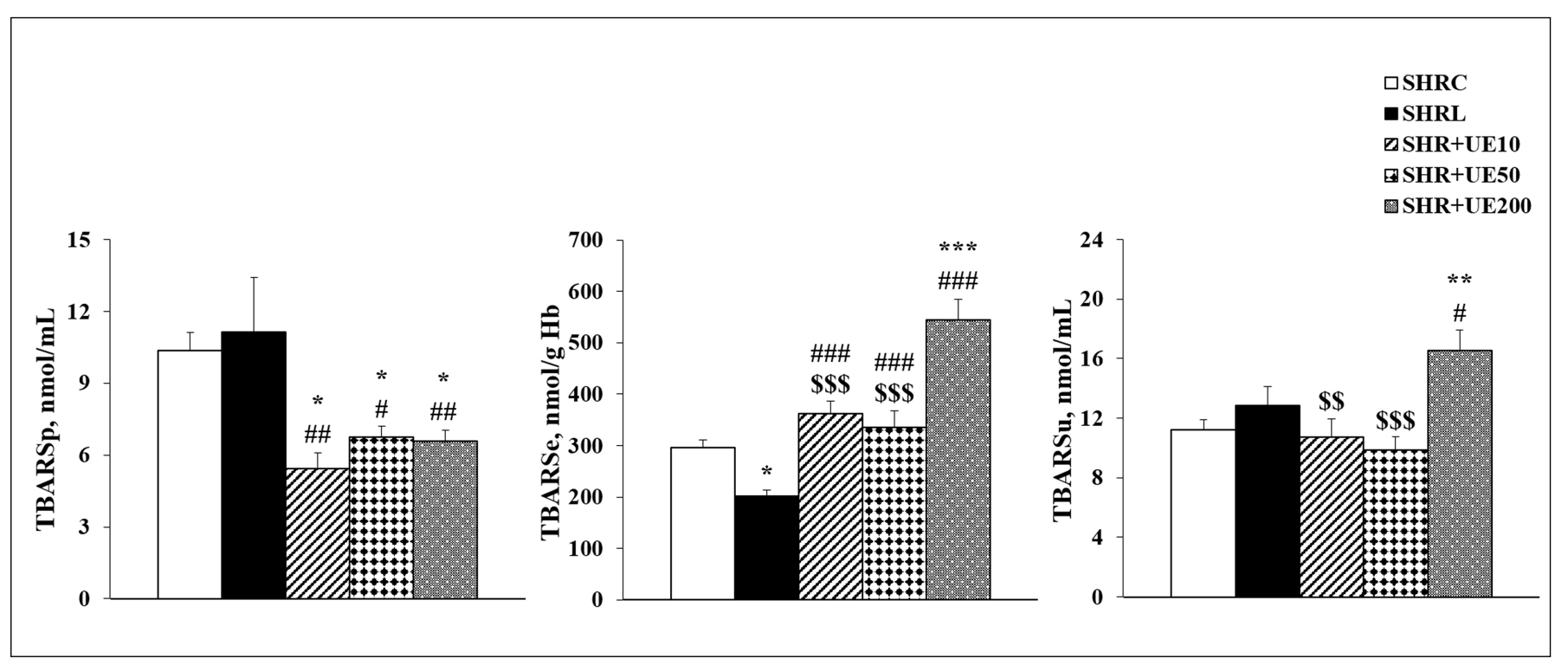

Fig. 4. Effects of four-week long daily supplementation with Urtica dioica L. leaf extract (UE) on lipid peroxidation in plasma (TBARSp), erythrocytes (TBARSe), and urine (TBARSu) of SHR (Published in Vajic et al., 2018); SHRC received $0.5 \mathrm{~mL} /$ day of water; SHR+L received 10 $\mathrm{mg} / \mathrm{kg} /$ day of losartan; SHR+UE10, SHR+UE50, and SHR+UE200 received 10, 50, and $200 \mathrm{mg} / \mathrm{kg} /$ day of UE, respectively; ${ }^{*} \mathrm{p}<0.05,{ }^{* *} \mathrm{p}<0.01$, and ${ }^{* * *} \mathrm{p}<0.001$ vs SHRC; \#p<0.05, \#\#p<0.01 and \#\#\# $<0.001$ vs SHR+L; $\$ \mathrm{p}<0.05, \$ \$ \mathrm{p}<0.01$, and $\$ \$ \$ \mathrm{p}<0.001$ vs SHR+UE200

through carotid, renal artery, and aorta. In addition, concentrations of nitric oxide metabolites (nitrites and nitrates) were measured in plasma and urine. Oxidative status was determined by oxidative stress by-products measurement (plasma, erythrocytes, and urine lipid peroxidation), measurement of antioxidant enzymes' activities and concentrations in erythrocytes (superoxide dismutase, catalase, glutathione peroxidase, and glutathione reductase), and by evaluating systemic antioxidative status (plasma antioxidant capacity). Standard biochemical parameters were measured as well, such as lipid status parameters (total cholesterol, LDL, HDL, triglycerides), and renal and liver function parameters. Expression of isoforms of nitric oxide synthases (eNOS, iNOS, and $\mathrm{nNOS}$ ) in the kidney was measured too.

\section{RESULTS}

The most abundant phenolic compounds in the Urtica dioica $\mathrm{L}$. leaf extracts were rutin, chlorogenic, and 2-O-caffeoyl malic acid (Fig.1). In addition, p-coumaroyl malate, caffeic acid, isoquercetin, kaempferol-3-O-rutinoside, isorhamnetin-3-O- rutinoside, and isorhamnetin hexoside have been also identified.

The preliminary phase of the optimization showed that aqueous-methanol had better extraction characteristics than aqueous-ethanol and water since aqueous-methanolic extracts had higher TP yield, as well as yield of individual phenolic compounds, and for this reasons, optimization was continued using aqueous-methanol. The next phase of optimization showed that the content of methanol in the solvent significantly influenced the yield of phenolic compounds in the extracts, but the extraction time did not. Results of RSM indicated that the highest yield of TP would be achieved using $54 \%$ aqueous-methanol and 38 extraction time (Fig.2). The application of ultrasound increased TP and TF yield, the content of chlorogenic and 2-O-caffeoyl malic acid, as well as the antioxidant capacities of the extracts, but decreased yield of rutin when compared to extracts prepared with maceration. The dry Urtica dioica L. leaf extract, obtained by the ultrasoundassisted extraction and optimal extraction parameters $(54 \%$ aqueous-methanol and 38 min extraction time), showed higher 


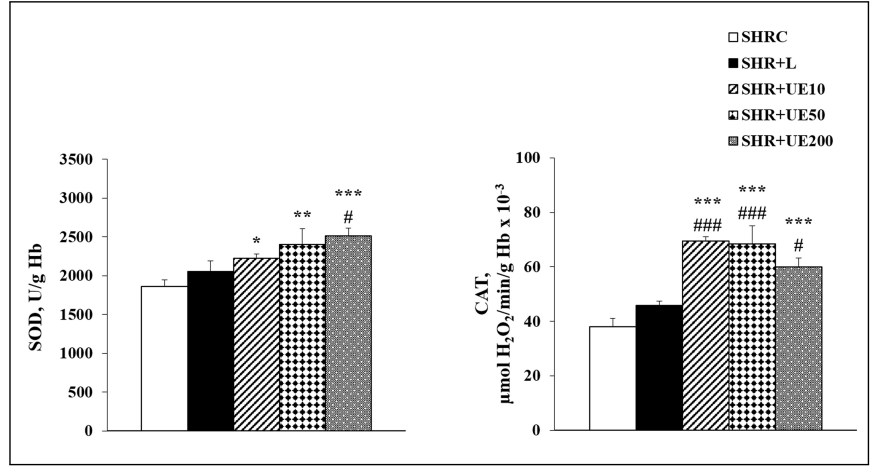

Fig. 5. Effects of four-week long daily supplementation with Urtica dioica L. leaf extract (UE) on activity of superoxide dismutase (SOD) and catalase (CAT) in erythrocytes of SHR (Published in Vajic et al., 2018) SHRC received $0.5 \mathrm{~mL} /$ day of water, SHR+L received $10 \mathrm{mg} / \mathrm{kg} /$ day of losartan, SHR+UE10, SHR+UE50, and SHR+UE200 received 10,50, and $200 \mathrm{mg} / \mathrm{kg} /$ day of UE, respectively ${ }^{*} p<0.05,{ }^{* *} p<0.01$, and ${ }^{* * *} p<0.001$ vs SHRC; $\# p<0.05$ and $\# \# \#$ p $<0.001$ vs SHR+L

antioxidant capacity than conventional antioxidant (BHT), but lower than vitamin C. Furthermore, UE showed good metal chelating ability.

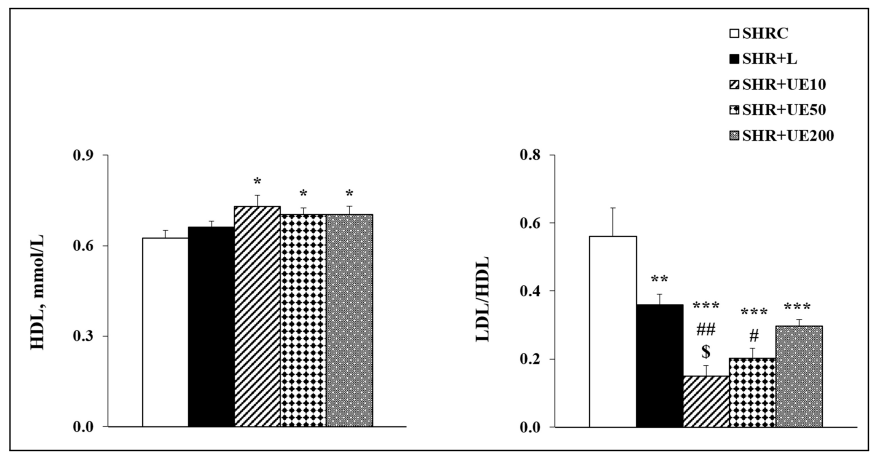

Fig. 6. Effects of four-week long daily supplementation with Urtica dioica L. leaf extract (UE) on concentration of HDL cholesterol and atherosclerosis-index (LDL/HDL) of SHR; SHRC received

$0.5 \mathrm{~mL} /$ day of water; $\mathrm{SHR}+\mathrm{L}$ received $10 \mathrm{mg} / \mathrm{kg} /$ day of losartan; SHR+UE10, SHR+UE50, and SHR+UE200 received 10, 50, and 200 $\mathrm{mg} / \mathrm{kg} /$ day of UE, respectively; ${ }^{*} \mathrm{p}<0.05,{ }^{* *} \mathrm{p}<0.01$, and ${ }^{* * *} \mathrm{p}<0.001$ vs SHRC; \#p $<0.05$ and $\# \# p<0.01$ vs $S H R+L ; \$ p<0.05$ vs SHR+UE200

Our in vivo study showed that four-week long oral treatment of SHR with 10, 50 and $200 \mathrm{mg} / \mathrm{kg}$ /day of UE decreased systolic and diastolic blood pressure, while treatment with 50 and $200 \mathrm{mg} / \mathrm{kg} /$ day of UE lowered the mean arterial pressure, too (Fig.3).Similarly, treatment with all three UE doses caused the decrease in cardiac output, but there were no changes in total peripheral vascular resistance and the heart rate, while stroke volume was decreased significantly only in the group treated with the highest UE dose. In addition, treatment with all three UE doses increased concentrations of nitrites and nitrates in plasma and urine of SHR. The oxidative status of SHR was improved upon treatment with all three UE doses as a result of the increased antioxidant capacity and decreased lipid peroxidation in plasma (Fig.4). In addition, activities of superoxide dismutase and catalase in erythrocytes was increased, as well (Fig.5). However, the treatment with $200 \mathrm{mg} / \mathrm{kg}$ per day of UE increased the concentration of lipid peroxidation products in erythrocytes and urine of SHR (Fig.4).

Treatment with all three UE doses increased plasma HDL concentration and lowered the parameters of atherosclerosis (Fig.6). Two higher UE doses caused an increase in the eNOS enzyme expression, while treatment with all three UE doses resulted in a decrease in the nNOS expression in the kidney of
SHR.

\section{CONCLUSION}

This dissertation shows that predominant phenolic compounds in Urtica dioica L. leaves are rutin, chlorogenic, and 2-O-caffeoyl malic acid. Optimization of the extraction parameters shows that maximal yield of phenolic compounds in the Urtica dioica L. leaf extracts will be achieved using $54 \%$ aqueous-methanol and $38 \mathrm{~min}$ long ultrasound-assisted extraction. In addition, extract obtained under these extraction conditions has high antioxidant activity. Four-week long treatment with Urtica dioica L. leaf extract has a beneficial effect on arterial pressure and systemic oxidative status in the experimental model of essential hypertension, promoting a dose of $10 \mathrm{mg} / \mathrm{kg} /$ day as an optimal and useful potential nutritional supplement for hypertensive subjects. Additionally, treatment with Urtica dioica L. leaf extract improves the lipid status of hypertensive subjects by increasing plasma HDL cholesterol and decreasing parameters of atherosclerosis. 